\title{
On the Influence of Chemical Defects and Structural Factors on Charge Transport and Failure in Polyethylene
}

\author{
S. Tantipattarakul ${ }^{1,2}$, A. S. Vaughan ${ }^{1}$ and T. Andritsch ${ }^{1}$ \\ ${ }^{1}$ Tony Davis High Voltage Laboratory. \\ University of Southampton, \\ Southampton, SO17 1BJ, UK \\ ${ }^{2}$ Electricity Generating Authority of Thailand, \\ Maintenance Division, \\ Nonthaburi, Thailand
}

\begin{abstract}
A blend of high and low density polyethylene was aged at $160^{\circ} \mathrm{C}$ in air and the impact of the chosen aging protocol on local chemistry, crystallinity and charge transport dynamics was considered. The aging conditions were chosen in order to exploit oxygen diffusion effects, such that the resulting systems could be considered as bi-layer specimens, containing two regions: a uniform lightly aged layer and a more spatially varying highly aged layer, which vary in the concentrations of aging-related defects such as carbonyl groups and unsaturation. For aging periods up to $3 \mathrm{~h}$, little space charge was found within both the highly and lightly aged layers. However, after aging for about $3.5 \mathrm{~h}$, an abrupt change in behavior was observed, whereby charges move rapidly through the highly aged layer, accumulating at the interface with the lightly aged layer. Sample melting behavior, as determined by differential scanning calorimetry, was found to depend on aging time, as a result of impeded crystallization and retarded reorganization kinetics. We suggest that this abrupt change in charge transport behavior is a consequence of the local concentration of chemically related trapping sites exceeding some critical threshold. The consequence of the resulting space charge distribution is a dramatic increase in the local electric field across the lightly aged layer and a consequent reduction in the overall DC breakdown strength. However, while further aging exacerbates these space charge effects, counter to expectations, the breakdown strength then recovers somewhat, suggesting a change in the underlying mechanism of electrical failure.
\end{abstract}

Index Terms — polyethylene, aging, space charge, dielectric breakdown

\section{INTRODUCTION}

CHARGE transport through disordered polymeric systems is of great scientific interest and technological importance. Scientifically, the issue has been addressed in a number of ways, including, theoretically, through simulation and by experiment. Early approaches to the evaluation of the band structure of polymers considered these as idealized infinite regular systems; in their 1971 paper, McAloon and Perkins [1], for example, contrasted the application of a linear combination of atomic orbitals molecular orbital (LCAO MO) approach with alternative published work and proposed a resulting band gap for polyethylene (PE) of around $19 \mathrm{eV}$, a value that is unreasonably large. The influence of the theoretical approach on the calculated band gap of a single, infinite polymer chain was discussed in detail by Süle et al [2], where band gap

Manuscript received on 17 August 2019, in final form 23 October 2019, accepted xx Month 20yy. Corresponding author: S. Tantipattarakul. energies ranging from 4.4 to $9.7 \mathrm{eV}$ were reported, depending on the methodology used. However, such an idealized structure differs greatly from the true nature of polymers. As a result, the impact of structural factors has attracted considerable interest, notably, through computational approaches. The impact of local ordering on the electronic structure of PE has been considered and band gap energy values of $6.2,6.7$ and $5.9 \mathrm{eV}$ were reported for amorphous, crystalline and interfacial structures respectively [3]. In addition to structural disorder, chemical factors also serve to create localized energy states (traps) near the band edges; an early study of the effect of carbonyl groups on the electronic properties of an oligomeric PE analogue suggested that carbonyl groups lead to the formation of trap states at a depth of $\sim 0.45 \mathrm{eV}$ [4]. Elsewhere, Wang et al [5] considered the impact of different chemical moieties (hydroxyl carbonyl and unsaturation) on the electronic structure of another oligomeric PE. This work indicated that the energy gap between the highest occupied and lowest unoccupied molecular 
orbitals was significantly reduced to $5.35 \mathrm{eV}$ in the case of their chosen conjugated structure.

Chemical species such as those described above are produced in $\mathrm{PE}$ as a result of degradation/aging. At elevated temperatures, chain scission generates free radicals, which may subsequently take part in a range of reactions. In the presence of oxygen, the formation of hydroperoxides is of particular importance, in that they can decompose, leading to branching reactions, further radical production [6] and the formation of ketones, esters, carboxylic acids and aldehydes [7]. Furthermore, Chabira et al [8] related vinyl group production in LDPE to the decomposition of ketones; the further reaction of such species can then facilitate crosslinking in $\mathrm{PE}$ at temperatures above the melting transition [9].

From the above, it is evident that the products of degradation in polymers markedly modify the band structure of such systems and, consequently, the effect of this on the electrical characteristics of technologically important insulation systems has been addressed. While macroscopic electrical degradation features, such as electrical trees, are reasonably wellunderstood, this is not the case for precursor phenomena, where a number of different processes have been suggested to lead to the modification of the material at the nanoscopic level. These include: chain scission at electric fields $>20 \mathrm{kV} / \mathrm{mm}$ and consequent formation of sub-microcavities causing local accumulation of space charge [10]; charge recombination and consequent electroluminescence leading to local degradation through photo-oxidation [11]; field-induced mechanical stress and consequent propagating cracking associated with breaking of inter-lamellar tie chains [12].

Nevertheless, whatever the precise mechanisms, while it is accepted that changes in local chemistry and microstructure are closely linked to modified electrical properties [13], the details are not fully understood. The work described here considers the effect of a specifically chosen aging protocol on the chemistry, lamellar texture and charge transport dynamics in a designed PE blend and, by considering structural factors, extends initial work reported recently [14]. The aging conditions were specifically chosen such that the local degradation reactions were kinetically controlled by oxygen diffusion into the system [15]. The rationale behind the choice of a PE blend stems from the ability to generate a wide range of different morphologies in a single system, while retaining a constant molecular composition [16]. Consequently, by varying the precise thermal history imposed on the system it is possible to position the chemical products of degradation at different locations within the system, on both the macroscopic and microscopic levels. Here, we focus on the former, where the specimen production route was chosen to provide as a simple morphology as possible [16]. Elsewhere, the effect of isothermal crystallization at 115 or $124{ }^{\circ} \mathrm{C}$ will be described in connection with different aging conditions, whereby comparable degradation products are "placed" into the overall system on a nanometric scale within the surrounding lamellar texture. In this way the interplay between local chemical effects, the spatial distribution of the crystal phase and charge transport dynamics is revealed.

\section{EXPERIMENTAL \\ 2.1 SAMPLE PREPARATION}

All samples were composed of a PE blend containing $20 \mathrm{wt} \%$ of high density PE (HDPE: HD5813EA, BP Chemicals) plus $80 \mathrm{wt} \%$ of low density PE (LDPE: LD100BW, Exxon Mobil), which was prepared by mixing the constituent components for 20 min at 160 ${ }^{\circ} \mathrm{C}$ in a Haake Polylab twin rotor R600 mixer. Samples, with a nominal thickness of $0.2 \mathrm{~mm}$, were then prepared by melt-pressing between sheets of polyethylene terephthalate (PET), before being quenched into distilled water [16].

Thermal aging was performed in a fan oven (Heraeus, Kendro Laboratory Products UT6) at $160^{\circ} \mathrm{C}$ for various ageing times, $t_{a}$, from 1 to $6 \mathrm{~h}$. These conditions were determined from initial scoping experiments, in which treatment temperatures from 120 to $160{ }^{\circ} \mathrm{C}$ and treatment times from $30 \mathrm{~min}$ to $24 \mathrm{~h}$ were explored. During the aging process, each film was placed with its lower surface in contact with a PET substrate and its upper surface exposed to air, in order to produce specimens, where the extent of aging varied through the thickness. Hereafter, the upper, more affected surface/layer is referred to as the highly aged surface/layer, while the lower less affected surface/layer is termed the lightly aged surface/layer. After aging, all samples were again quenched directly into distilled water, in order to generate as simple a lamellar morphology as possible.

\subsection{MOLECULAR CHARACTERIZATION}

The spatial variation of aging-induced chemical changes was determined by confocal Raman microprobe spectroscopy. To prepare the required sample cross-sections, the aged film of interest was sandwiched between two sheets of a styrene, ethylene/butylene, styrene triblock copolymer (Kraton G1650), which had previously been softened through exposure to toluene. After evaporation of the toluene, the resulting layered sample was microtomed at $-40{ }^{\circ} \mathrm{C}$ using an RMC MT7, CR-21 cryo-ultramicrotome. Raman analysis was then performed using a Renishaw RM1000 system with a Renishaw NIR 780TF diode laser (wavelength $780 \mathrm{~nm}$ and maximum output power of $25 \mathrm{~mW}$ ). Data were acquired with a $\mathrm{x} 50$ objective lens and with the laser power set at $50 \%$, from $500 \mathrm{~cm}^{-1}$ to $3200 \mathrm{~cm}^{-1}$, using 10 consecutive $10 \mathrm{~s}$ extended scans. The resulting data were processed to remove background scattering and normalized with respect to the Raman peak at $1295 \mathrm{~cm}^{-1}$ (C-C twisting mode) [17]. Carbonyl index (CI) values were determined using:

$$
C I=A_{c} / A_{\text {ref }}
$$

where $A_{c}$ and $A_{\text {ref }}$ are, respectively, the areas under the peaks at $1720 \mathrm{~cm}^{-1}(\mathrm{C}=\mathrm{O}$ stretching $)$ and around $2850 \mathrm{~cm}^{-1}\left(\mathrm{CH}_{2}\right.$ and $\mathrm{CH}_{3}$ symmetric and asymmetric stretching).

Complementary spectral data were obtained from highly aged surfaces using attenuated total internal reflectance (ATR) Fourier transform infrared spectroscopy (FTIR). For this, a Thermo Scientific Nicolet iS5 FT-IR spectrometer with iD7 ATR diamond crystal plates was used; all spectra were obtained over the wavenumber range $400-4000 \mathrm{~cm}^{-1}$ and, for each spectrum, 16 scans were averaged with $4 \mathrm{~cm}^{-1}$ resolution. 
While the above approaches provide information on the effect of aging at the functional group level, they do not readily reveal changes in molecular topology. As such, gel content measurements were also conducted using xylene extraction at $140{ }^{\circ} \mathrm{C}$ for $6 \mathrm{~h}$, in accordance with ASTM D2765-01. The gel content was calculated as the ratio of the dried insoluble residue to the initial sample mass (typically, $\sim 0.3 \mathrm{~g}$ ).

\subsection{MORPHOLOGY}

Different scanning calorimetry (DSC) (Perkin Elmer DSC 7, running Pyris software) was used to determine the melting behavior of each specimen. All experiments were performed after calibration of the DSC with high-purity indium. All sample melting data were acquired from $40^{\circ} \mathrm{C}$ to $170{ }^{\circ} \mathrm{C}$ at a scan rate of $10^{\circ} \mathrm{C} / \mathrm{min}$ using the first heating scan after sample production by quenching. Subsequent data analysis was conducted using the Origin 2018 software package.

\subsection{ELECTRICAL PROPERTIES}

Electrical properties were determined at room temperature $\left(20^{\circ} \mathrm{C}\right)$ using the following techniques. The accumulation and dissipation of space charge (SC) was examined using the pulsed electro-acoustic (PEA) method, using a semiconducting composite (semicon) anode (high voltage) and an aluminum cathode (ground potential). For this, an applied voltage of $8 \mathrm{kV}$ was chosen and data were, first, acquired for $3600 \mathrm{~s}$ with the field applied (voltage-on) and, subsequently, with the sample short-circuited (voltage-off). In view of the inherent asymmetry in the aged samples, experiments were performed with the highly aged surface in contact with the anode and with the highly aged surface in contact with the cathode.

DC breakdown strength was measured according to ASTM D3755-14. For this, the sample was immersed in silicone oil between spherical electrodes, $6.3 \mathrm{~mm}$ in diameter, and the applied voltage increased at a rate of $350 \mathrm{~V} / \mathrm{s}$. Breakdown voltage and sample thickness data were collected from twenty breakdown sites per sample and the resulting breakdown fields were analyzed assuming two parameter Weibull statistics with $90 \%$ confidence bounds, using the Origin 2018 software.

\section{RESULTS}

\subsection{AGING-INDUCED PHYSICOCHEMICAL CHANGES}

Figure 1 shows the effect of the chosen aging regime on the time evolution of $\mathrm{CI}$ at different locations within the samples. Carbonyl formation occurs through chain scission followed by reaction with available oxygen $[7,15]$ and, therefore, $\mathrm{CI}$ is used here as a simple proxy for the extent of aging. Consider, first, the data obtained at $130-190 \mu \mathrm{m}$ below the uppermost highly aged surface, where CI increases progressively with aging time. Conversely, at depths from zero to $100 \mu \mathrm{m}$, the Raman data evince a marked increase in the rate of oxidation beyond an induction period of $2-3 \mathrm{~h}$. Thus, initially, aging in air at 160 ${ }^{\circ} \mathrm{C}$ for times up to $2 \mathrm{~h}$ has relatively little effect on the measured carbonyl index at any point within the specimens which, we suggest, is related to local consumption of the included antioxidant [6]. The materials used here were both commercial

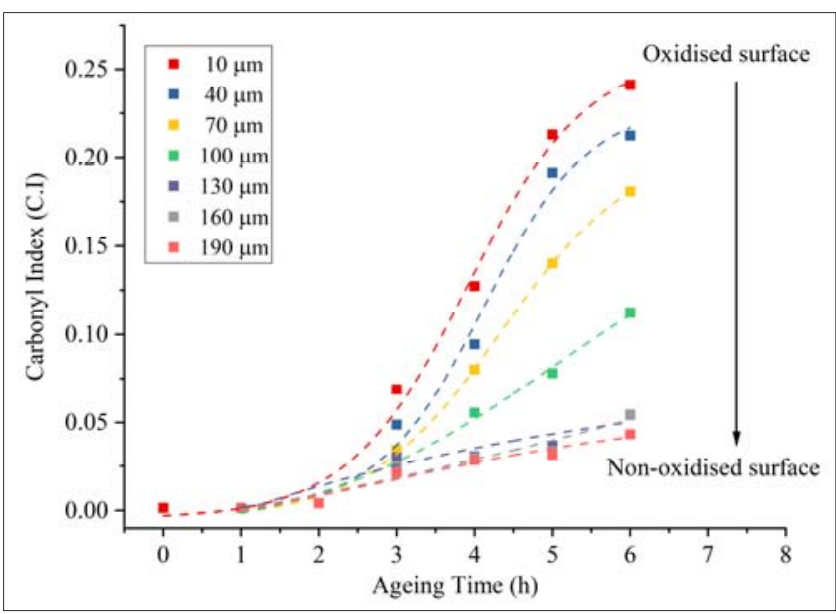

Figure 1. Confocal Raman data presented in the form of carbonyl index as a function of aging time at different positions within sample cross-sections.

systems and, therefore, would be expected to include relevant protection packages. We did seek direct evidence for both the initial presence of the antioxidant and for its depletion, but were unsuccessful on sensitivity grounds. Nevertheless, the observed behavior is consistent with published work [18] and, therefore, we believe that the above interpretation is viable. Thereafter, for $t_{a}>2 \mathrm{~h}$, the observed position dependent increase in the CI value is associated with the local degree of autoxidation, which is driven primarily by the availability of oxygen at each location within the sample. Audouin et al [15] described this in terms of diffusion-limited effects, whereby an oxidized "layer" is formed of approximate thickness $(D / k)^{0.5}$, where $D$ is the diffusion coefficient and $k$ is the pseudo-first-order reaction rate constant for oxygen consumption. In summary, aging occurs non-uniformly throughout the sample and, as described above, can be considered in terms of a highly aged layer, within which the degree of oxidation varies with position. From Figure 1, this ranges from the uppermost sample surface to a depth between 100 and $130 \mu \mathrm{m}$. Below this, the material is relatively lightly and more uniformly aged.

Figure 2 shows complementary data obtained by FTIR from the uppermost highly aged surface of samples aged for different times. These show that the strength of carbonyl-related absorption bands, including ketones and carboxylic acids (at $1716 \mathrm{~cm}^{-1}$ ), esters and aldehyde (at $1725 \mathrm{~cm}^{-1}$ ) and $\gamma$-lactone (at $1780 \mathrm{~cm}^{-1}$ ), increase significantly with aging time beyond $2 \mathrm{~h}$. As such, these data mirror the above Raman data. While the use of CI as a general proxy for the extent of aging is not unreasonable, it does fail to recognize all of the products of thermal treatment. Indeed, Figure 2 shows the presence of esters (1264 and $\left.1289 \mathrm{~cm}^{-1}\right)$, ethers $\left(1170,1112\right.$ and $\left.1134 \mathrm{~cm}^{-1}\right)$ alcohols (1012 and $\left.1046 \mathrm{~cm}^{-1}\right)$, vinyl groups (vinylidenes at $874 \mathrm{~cm}^{-1}$, vinyls at $903 \mathrm{~cm}^{-1}$ ) and hydroxyl groups (over 3,000$\left.3,600 \mathrm{~cm}^{-1}\right)[8,19]$. On aging for more than $2 \mathrm{~h}$, the intensity of the majority of these bands increase significantly, while the intensity of absorption bands related to hydroxyl groups (over $3,000-3,600 \mathrm{~cm}^{-1}$ ) remains close to constant. While variations in the carbonyl group concentration can, as above, be used as a chemical indicator of extent of aging, Chabira et al [8] have 


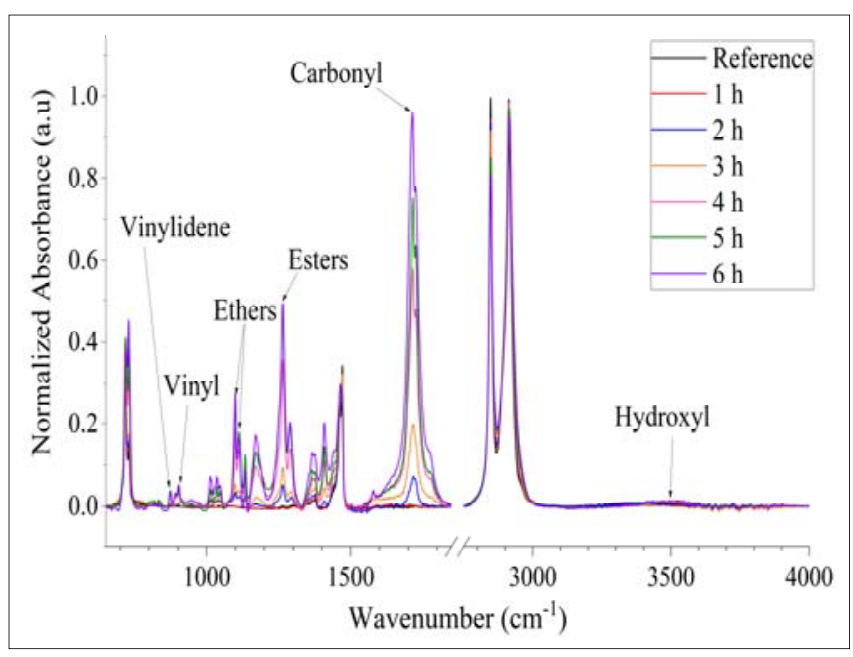

Figure 2. The IR spectra obtained from the highly aged surface of the samples with respect to the variation of aging times from 0 to $6 \mathrm{~h}$.

suggested that vinyl groups can be used as complementary chemical indicators of chain scission processes. Specifically, these workers have associated the formation of vinylidenes to $\beta$-scission of branched (tertiary) alkyls abstracted from tertiary carbon in PE chains, while vinyl groups arise from Norrish type II-scission of ketones [8, 19]. Additionally, Johnston et al [9] reported that cross-linking becomes the dominant process in place of chain scission when polyethylene experiences high degrees of oxidative degradation at a high temperature; for this reason, gel fraction measurements were undertaken.

Figure 3 shows the effect of aging time on gel content. Up to $2 \mathrm{~h}$ aging time, negligible gel was formed while, beyond this, the measured gel content increased progressively, in a comparable manner to the changes seen in CI within the highly aged layer. Crosslinking during the thermo-oxidative aging of polyethylene was described by Ranby and Rabek [20] and related to reactions between alkoxy radicals ( $\mathrm{R}-\mathrm{CO} \bullet-\mathrm{R})$ or between alkoxy and alkyl radicals, leading to the formation of esters and ethers respectively. As such, the existence of strong IR absorbance in the ester and ether regions for samples aged beyond $2 \mathrm{~h}$ may be associated with such crosslinking processes. Elsewhere, it has been suggested that crosslinks may also form through the linkage of alkyl radicals $\left(\mathrm{R}-\bullet \mathrm{CH}_{2}-\mathrm{R}\right.$; secondary and tertiary alkyls) or vinyl groups and alkyl radicals [19]. The presence of vinylidenes and vinyl groups indicates that such processes are viable here, while their low concentration may be a consequence of their consumption through crosslinking [8, 19].

In summary, data shown above present a consistent picture of the effects of the chosen aging conditions, which include the formation of molecular defects ranging from discrete chemical species to modified molecular topologies. Specifically, the extent of degradation varies through the cross-section of the aged samples, which can be considered to consist of highly aged and lightly aged layers.

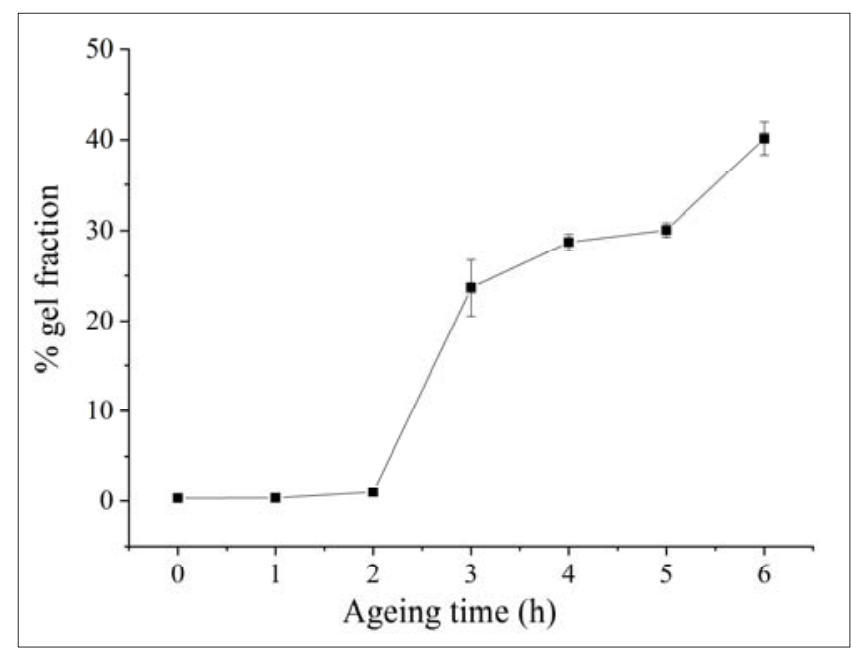

Figure 3. The percentage of crosslinks in the samples with the variation of aging times.

\subsection{STRUCTURAL CONSEQUENCE OF AGING}

The aging-related changes described above can be considered as introducing molecular defects into the system, which could affect its ability to crystallize. Figure 4 presents DSC data obtained from samples aged for the times indicated. Consider first the behavior of the unaged sample which, on heating in the

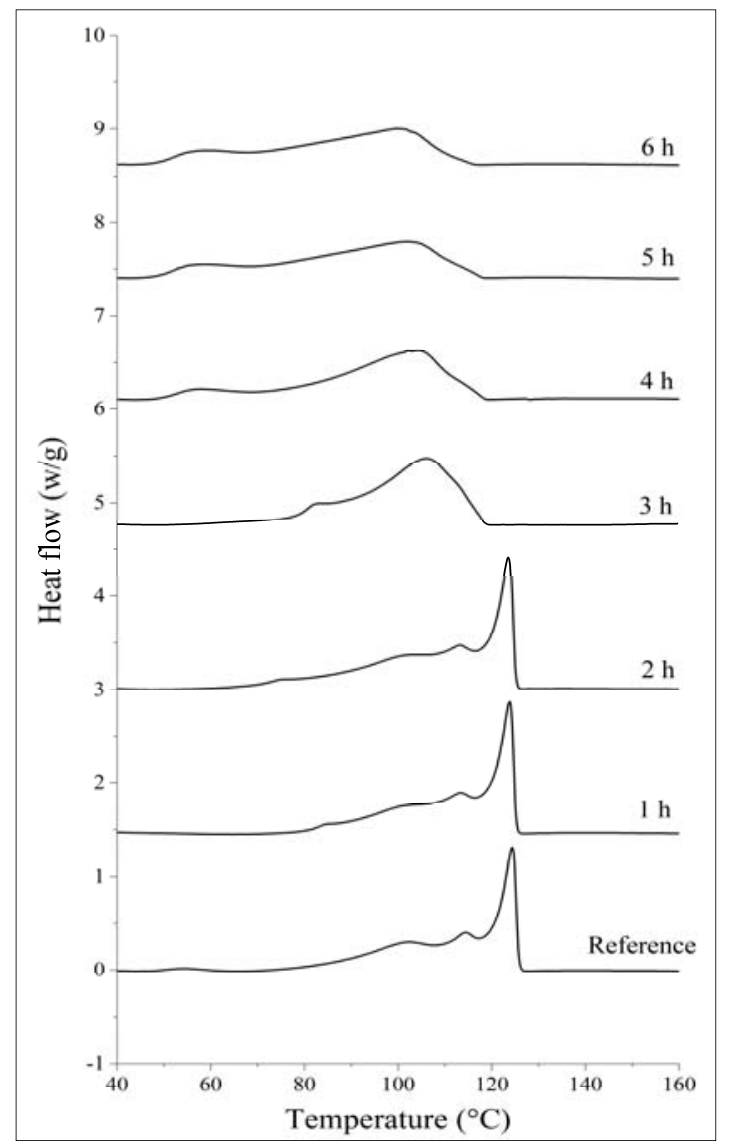

Figure 4. DSC melting traces of the samples with the variation of aging times from 0 to $6 \mathrm{~h}$. 


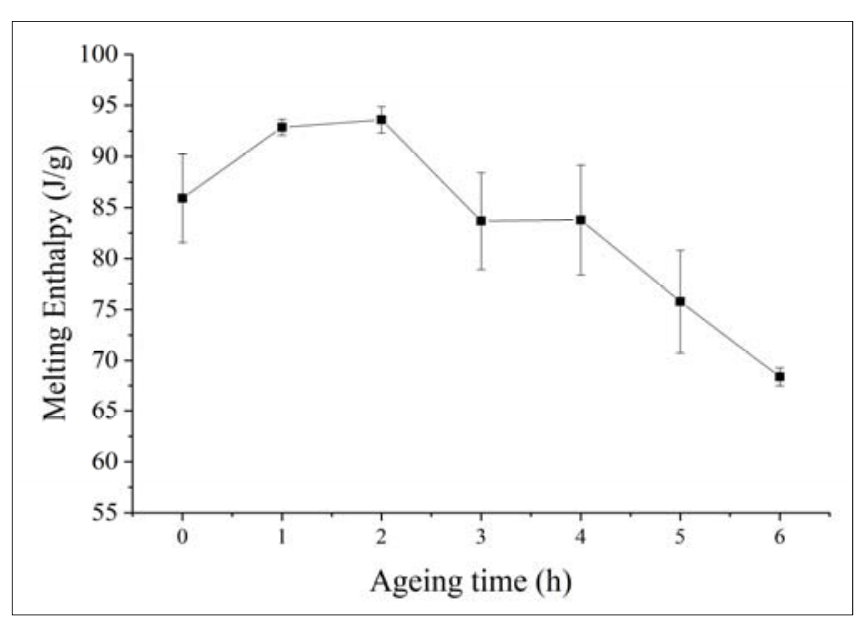

Figure 5. Variation in total melting peak enthalpy with aging.

DSC, exhibits a broad melting endotherm that spans the range from 60 to $125^{\circ} \mathrm{C}$ and which contains three features. First, there is a well-defined peak (maximum at $\sim 124{ }^{\circ} \mathrm{C}$ ), which relates to a lamellar population composed principally of HDPE. The location of this peak is a consequence of a fraction of the initially formed lamellar population, coupled with subsequent thickening during the DSC scan [21]. The next melting feature (minor peak, maximum at $\sim 115{ }^{\circ} \mathrm{C}$ ) results from cocrystallization of HDPE and the more linear molecular sequences from within the LDPE; as a consequence of the latter, annealing during the DSC scan is suppressed, hence the intermediate location. Finally, the broad melting transition that extends down in temperature to $\sim 60{ }^{\circ} \mathrm{C}$ is associated with the melting of crystals formed from the remnant, more highly branched LDPE fraction [16].

At low aging times $(0-2 \mathrm{~h})$, the Raman data presented in Figure 1 indicate that the system is chemically homogeneous and, therefore, the melting behavior of these systems should be uniform. While the overall form of the melting endotherm is retained, Figure 5 indicates that the molecular changes that result from aging within the induction period lead to a slight increase in crystallinity. This behavior is consistent with a number of studies of thermal aging effects in PE [22], where it has been related to the recrystallization of segments of abstracted and disordered PE chains in amorphous regions. For longer times, the imposed aging protocol results in systems that are increasingly spatially differentiated in terms of degradation products, such that the observed behavior should reflect contributions from both the highly and lightly aged layers. Consider first, the sample aged for $3 \mathrm{~h}$ exhibits a markedly changed melting endotherm spanning the temperature range $60-120^{\circ} \mathrm{C}$. From above, it is clear that such samples contain a higher concentration of aging-induced chemical defects and a measurable gel fraction $>20 \%$. As such, we interpret the marked difference in the form of the melting endotherm as being a consequence of two factors: aging-induced defects acting to limit the initial crystallization of the system; reduced dynamic reorganization during the DSC heating scan [23]. These effects will be most severe in the most highly aged region of the specimen $(C I=0.24)$ but, evidently, even the lightly aged region is also affected $(C I=0.043)$. As ageing progresses further, the overall crystallinity (see Figure 5) falls monotonically; while some features within the melting endotherm are retained (the peak maximum remains constant at $101 \pm 1.37{ }^{\circ} \mathrm{C}$ ), this is accompanied by a progressive displacement of the lowest melting lamellar populations to lower temperatures. In toto, we interpret this as being, primarily reflective of increasingly severe degradation within the highly aged layer of each sample, while recognizing that even the lightly aged layer is not unaffected. As discussed above, the notion of two distinct and sharply differentiated regions is, of course, an approximation. Nevertheless, even after $6 \mathrm{~h}$ aging, the Raman CI at the lightly aged surface is 0.043 , which is comparable to that at the center of the sample after just $3 \mathrm{~h}$ at $160^{\circ} \mathrm{C}$.
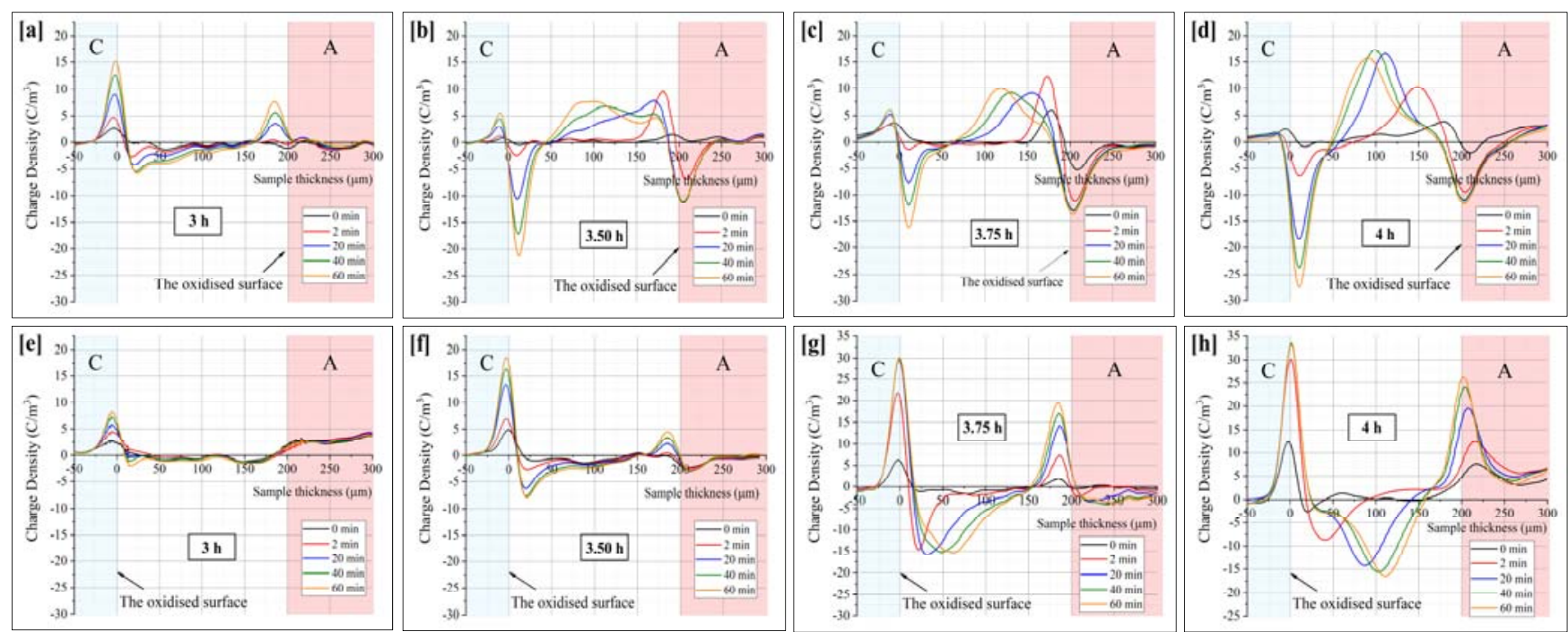

Figure 6. Charge accumulation profiles (voltage-on) obtained from samples aged for between $3 \mathrm{~h}$ and $4 \mathrm{~h}$ with the highly aged surface in contact with: (a-d) the semiconducting composite high voltage anode $(\mathrm{A})$ and $(\mathrm{e}-\mathrm{h})$ the aluminum earth potential cathode $(\mathrm{C})$. 


\subsection{AGING AND CHARGE TRAPPING}

Elsewhere [14], we have presented a general overview of aging and space charge accumulation in a quenched HDPE/LDPE and, therefore, we will focus here on specimens aged from $3 \mathrm{~h}$ to $4 \mathrm{~h}$, where dramatic changes occur. For the traces presented in Figure 6, the charge on the electrodes has been subtracted from the raw data in order better to reveal the space charge within the polyethylene and the consequent induced charges on the electrodes. From Figure $6 \mathrm{a}$ it is clear that little space charge accumulates within the bulk of the sample aged for $3 \mathrm{~h}$, space charge profiles indicating the accumulation of a small amount of negative homocharge within the lightly aged layer of the specimen adjacent to the cathode (to the left of the plots). This occurs in spite of the molecular changes revealed above that occur on aging for periods up to 3 h. In contrast, the data presented in Figure $6 \mathrm{~b}$ reveal that after aging for $3.5 \mathrm{~h}$, positive charge is injected from the anode (to the right of the plots) and, over a period of about $40 \mathrm{~min}$, moves progressively through the sample towards the cathode. Further aging results in an increase in the density of space charge and more rapid movement (see Figures $6 b-6 d$ ). Data obtained with the sample geometry reversed are presented in Figures 6e to 6h; that is, with the more highly aged surface adjacent to the cathode (to the left of the plots). Again, in the sample aged for $3 \mathrm{~h}$, only a relatively small amount of homocharge is evident within the lightly aged layer within the specimen; after $3.5 \mathrm{~h}$ of aging, negative homocharge can be seen to be injected from the cathode into the highly aged layer, where it is trapped locally. Increasing the aging time by just 15 min serves dramatically to increase both the amount of negative injected space charge and its rate of movement through the highly aged layer towards the anode.

While data obtained with the two sample configurations are broadly comparable, Figure 6 indicates that the transition from the accumulation of a small quantity of homocharge within the lightly aged layer, to the injection and propagation of large amounts of charge through the highly aged layer is displaced to longer times, when the highly aged surface is in contact with

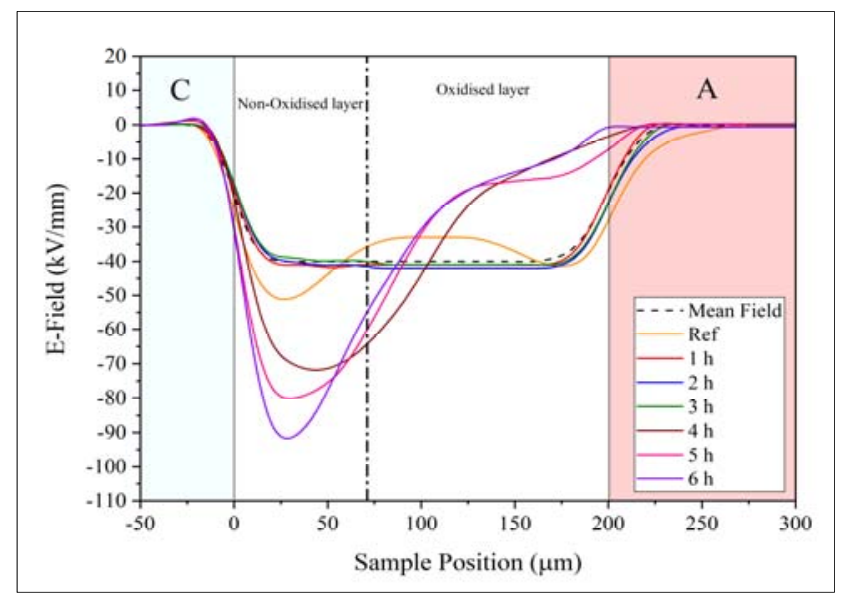

Figure 7. Spatial variation in electric field through the sample thickness as a consequence of accumulated space charge. Anode in contact with the highly aged surface. the aluminum cathode. Multiple experimental repeats showed this to be reproducible and, therefore, we suggest that it is a genuine effect that is related either to the nature of the dominant charge carrier under different polarities, or associated with differences in charge injection/extraction from the semiconducting composite (SC) anode and an aluminum (Al) cathode. Such effects have been addressed in a number of studies. Wang et al [24] used density functional theory (DFT) to demonstrate that positive charge injection and accumulation are dominated by the low hole injection barrier in LDPE, compared with electron injection. Chen, et al [25], studied the effects of electrode material and polarity on space charge formation in polyethylene and showed that the use of a semiconducting composite electrode leads to increased charge injection and space charge accumulation in polyethylene, compared to an $\mathrm{Al}$ electrode. This effect was reported to be independent of the applied polarity, suggesting that the nature of the charge carrier is less important than the interfacial potential barrier for injection. The results presented in Figure 6 indicate the early onset of positive charge injection from the semiconducting composite anode and, as such, are consistent with both the theoretical and experimental studies described above.

\subsection{AGING, CHARGE TRANSPORT AND BREAKDOWN}

The accumulation of space charge within a dielectric directly affects the distribution of the electric field within the system and, therefore, this issue was considered for the case of the anode in contact with the highly aged surface (see Figure 7). In the unaged sample, heterocharge accumulation adjacent to the cathode results in a local increase in the local electric field at this location to about $50 \mathrm{kV} \mathrm{mm}^{-1}$. In the case of samples aged for up to $3 \mathrm{~h}$, the replacement of this heterocharge with the small amount of homocharge seen in Figure 6, leads to the maximum local electric field determined from the space charge plots being constant at $37-41 \mathrm{kV} \mathrm{mm}^{-1}$, as expected for Laplacian fields in this experimental arrangement. However, for $t_{a}=4 \mathrm{~h}$, the marked change in the space charge profile discussed above

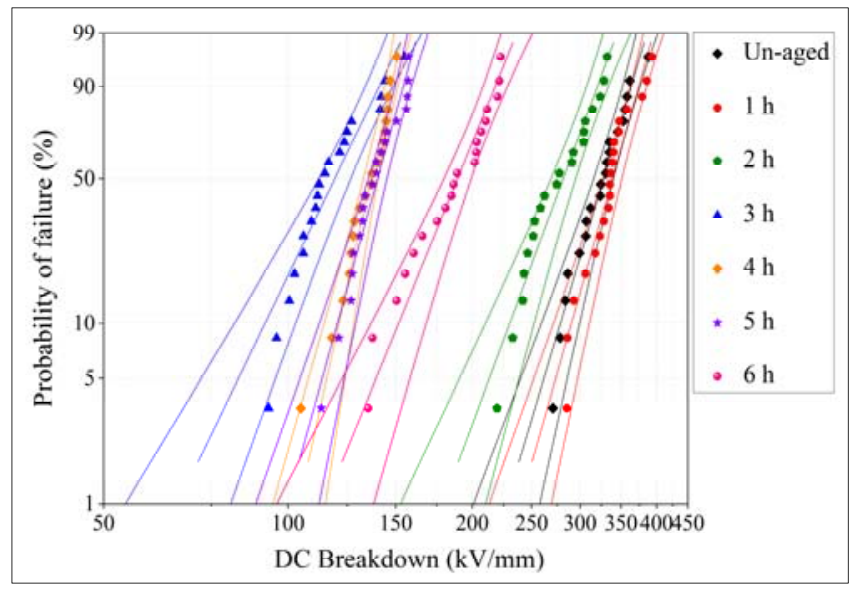

Figure 8. Weibull plot for the dielectric breakdown strength of the reference sample and aged samples in case of the highly aged surface in contact with the cathode. 


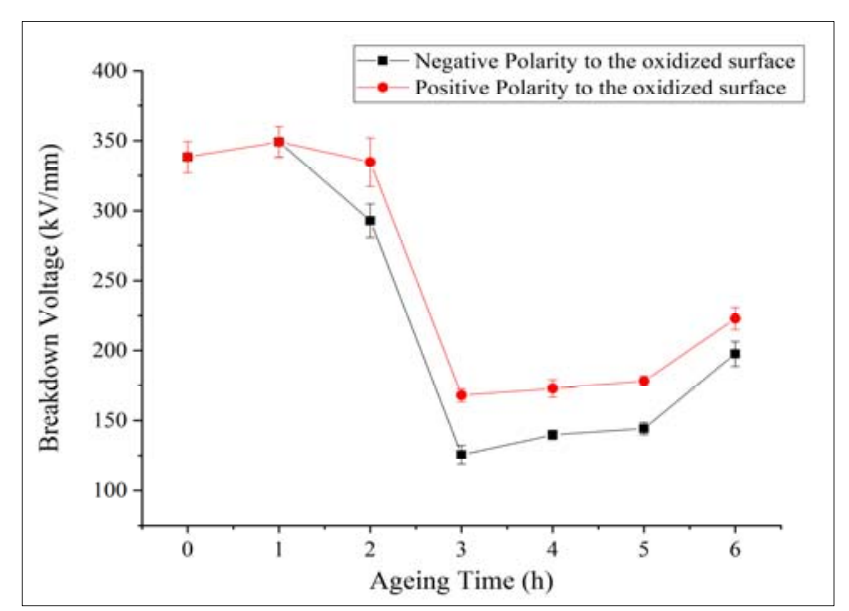

Figure 9. DC breakdown strength data obtained with the highly aged surface in contact with the anode and cathode electrodes.

results in a highly non-uniform field distribution within the specimen, such that the electric field is strongly intensified within the lightly aged layer (maximum value $>90 \mathrm{kV} \mathrm{mm}^{-1}$ ).

Figure 8 shows representative DC breakdown data presented in the form of Weibull plots, while Figure 9 summarizes such behavior in terms of the derived Weibull scale parameter, $\alpha_{t a}$. First, comparison of data obtained with the anode and the cathode in contact with the highly aged surface reveals reduced breakdown strength values with the latter arrangement (negative polarity) for $t_{a}>1 \mathrm{~h}$. While the presence of asymmetry parallels the different ageing times required for the onset of extensive space charge accumulation seen in Figure 6, it is not intuitively obvious why the geometry corresponding to suppressed space charge accumulation leads to consistently reduced values of $\alpha_{t a}$. Furthermore, both of the data sets presented in Figure 9 can, broadly, be considered in terms of two regimes, which are characterized by very different breakdown strength values. For example, compare results obtained with the anode in contact with the highly aged surface, where $\alpha_{2}$ equates to $335 \pm 17 \mathrm{kV} / \mathrm{mm}$ and $\alpha_{4}=173 \pm 6 \mathrm{kV} / \mathrm{mm}$. That is, the latter value is only about $50 \%$ of the former. From Figure 7 , the peak internal field within the specimen, based upon the space charge profiles acquired with $8 \mathrm{kV}$ applied across samples nominally $200 \mu \mathrm{m}$ in thickness with the anode in contact with the aged surface, is $42 \mathrm{kV} / \mathrm{mm}$ in the sample aged for $2 \mathrm{~h}$ and $72 \mathrm{kV} / \mathrm{mm}$ in the sample aged for $4 \mathrm{~h}-\mathrm{a}$ field intensification of some $58 \%$.

Contrast now samples aged for between $3 \mathrm{~h}$ and $6 \mathrm{~h}$ and, as above, consider data acquired with the highly aged surfaces in contact with the anode. From Figure 7, the peak internal field based upon the PEA data rises monotonically from $40 \mathrm{kV} / \mathrm{mm}$ to $92 \mathrm{kV} / \mathrm{mm}$ with increasing aging time. In parallel, from Figure 9, the overall measured breakdown strength increases from $\alpha_{3}=168 \pm 5 \mathrm{kV} / \mathrm{mm}$ to $\alpha_{6}=223 \pm 8 \mathrm{kV} / \mathrm{mm}$, despite the marked field intensification suggested by the space charge profiles.

For numerous reasons it could be argued that is inappropriate to compare PEA and breakdown data too closely. Breakdown occurs in the range $100-400 \mathrm{kV} / \mathrm{mm}$; the experimental durations are significantly different; the PEA data were acquired using semicon and aluminum electrodes, whereas breakdown experiments were conducted between opposing steel ball bearing electrodes. Nevertheless, we suggest that the behavior reported above is worthy of collective discussion. For example, in the case of the specimens aged for $3-4 \mathrm{~h}$, the duration of a breakdown experiment is $\sim 100 \mathrm{~s}$; from the data presented in Figure 6, little space charge then accumulates within the specimen in these less heavily aged specimens. Conversely, in the sample aged for $6 \mathrm{~h}$, space charge accumulates rapidly [14] and would therefore be expected to promote breakdown, which is contrary to what is seen. The implication of this is therefore that the measured breakdown strength is not dominated by the accumulated space charge but, rather, results from a combination of space charge effects and variations in material properties. In this regard, it is worth returning at this point to Figure 3 and 5; from the former, the gel content increases with increased aging times in the range 3 $-6 \mathrm{~h}$ while overall crystallinity decreases. This implies that in the systems considered here, which were deliberately prepared so as to suppress extensive lamellar development, morphology per se is of little importance in determining breakdown strength, whereas possible variations in mechanical properties that result from the presence of an increasing gel fraction may be significant. Indeed, optical examination of failure regions in samples aged from 3 to $6 \mathrm{~h}$ was highly suggestive of mechanically-related variations in the final failure.

\section{CONCLUSIONS}

The work described above reports on effects of aging on structural and electrical factors in PE, where the spatial distribution of ageing-induced moieties has been controlled by exploiting diffusion-limited oxidation process such that the resulting specimens can be considered in terms of highly and lightly aged layers. For aging times up to $2 \mathrm{~h}$ at $160^{\circ} \mathrm{C}$, analysis by confocal Raman spectroscopy, FTIR spectroscopy, DSC and the measurement of gel content, reveals few material changes, which we attribute to the stabilizing effect of the included antioxidant. Within this ageing regime, minimal space charge accumulation occurs and the measured DC breakdown strength remains close to that measured for the unaged system. Beyond about $3 \mathrm{~h}$, the chemical and structural consequences of aging increase progressively and monotonically, as represented by indicators such as carbonyl index, gel content and crystallinity. In contrast, abrupt and marked changes in space charge behavior were seen. These are consistent with space charge limited conduction (SCLC) effect, whereby low concentrations of chemical changes equate to a relatively low density of deep traps while, beyond some threshold, the increased density of comparable moieties serves to facilitate rapid migration of space charge through the highly aged layer, resulting in intensification of the local field within the lightly aged layer. However, quantitative comparison of the degree of field intensification and the measured breakdown strength indicate poor agreement. Indeed, for aging times beyond $3 \mathrm{~h}$ under the conditions described here, increases in DC breakdown strength with increasing ageing times can be observed in samples where field intensification from space charge is increasingly severe. As such, we suggest that breakdown in such specimens cannot be solely related to space charge effects and related field 
intensification, but suggest that, in parallel, certain aspects of the aging process are serving to reinforce the material, leading to the observed increases in breakdown strength with increased degrees of aging.

\section{ACKNOWLEDGMENT}

The authors acknowledge the Electricity Generating Authority of Thailand and the University of Southampton for financial sponsorship. All data supporting this study are openly available from the University of Southampton repository at https://doi.org/10.5258/SOTON/D1047.

\section{REFERENCES}

[1] B. J. McAloon and P. G. Perkins, "Semi-empirical LCAOMO theory for infinite systems. Part 1.-Application to polyethylene, polyacetylene and polyvinylchloride," J. Chem. Soc., Faraday Trans. 2., vol. 68, pp. 1121-1132, 1972.

[2] P. Süle, S. Kurth, and V. E. Van Doren, "Orbital dependent exchangeonly methods for periodic systems," Phys. Rev. B, vol. 60, no. 8, pp. 5429-5439, 1999.

[3] M. Unge, T. Christen, and C. Törnkvist, "Electronic structure of polyethylene - Crystalline and amorphous phases of pure polyethylene and their interfaces," Annu Rep. Conf. Electr. Insul. and Dielect. Phenom. (CEIDP), 2012, pp. 525-530.

[4] M. Meunier, N. Quirke, and A. Aslanides, "Molecular modeling of electron traps in polymer insulators: Chemical defects and impurities," J. Chem. Phys., vol. 115, no. 6, pp. 2876-2881, 2001.

[5] W. Wang, Y. Tanaka, and T. Takada, "Space charge of polyethylene and electronic structure analysis of trapping site using common chemical groups," Sens. and Mater., vol. 29, no. 8, pp. 1223-1231, 2017.

[6] A. Peacock, Handbook of Polyethylene: Structures, Properties, and Applications, Marcel Dekker, Inc., 2000.

[7] F. Gugumus, "Physico-chemical aspects of polyethylene processing in open mixers1: Review of published work," Polym. Degrad. Stab., vol. 66, pp. 161-172, 1999.

[8] S. F. Chabira, M. Sebaa, and C. G'Sell, "Oxidation and crosslinking processes during thermal aging of low-density polyethylene films," J. Appl. Polym. Sci., vol. 124, pp. 5200-5208, 2011.

[9] R. T. Johnston, E. J. Morrison, and R. Clough, "Thermal scission and cross-linking during polyethylene melt processing," Adv. Chem., vol. 249, pp. 651-682, 1996.

[10] J.-P. Crine, "Influence of high electrical fields on aging and polarization properties of polyethylene," Polym. Int., vol. 51, pp. 1159-1163, 2002.

[11] C. Laurent, G. Teyssedre, S. L. Roy, and F. Baudoin, "Charge dynamics and its energetic features in polymeric materials," IEEE Trans. Dielectr. Electr. Insul., vol. 20, no. 2, pp. 357-377, 2013.

[12] J. P. Jones, J. P. Llewellyn, and T. J. Lewis, "The contribution of fieldinduced morphological change to the electrical aging and breakdown of Polyethylene," IEEE Trans. Dielectr. Electr. Insul., vol. 12, no. 5, pp. 951-966, 2005

[13] A. Tzimas, S. M. Rowland, and L. A. Dissado, "Effect of electrical and thermal stressing on charge traps in XLPE cable insulation," IEEE Trans. Dielectr. Electr. Insul., vol. 19, no. 6, pp. 2145-2154, 2012.

[14] S. Tantipattarakul, A. S. Vaughan, and T. Andritsch, "On the influence of morphology and chemical defects on charge transport dynamics in polyethylene: thermal aging and concentration gradient," J. Phys. D: Appl. Phys., vol. 52, no. 39, pp. 1-14, 2019.

[15] L. Audouin, V. Langlois, J. Verdu, and J. C. M. d. Bruijn, "Role of oxygen diffusion in polymer aging: kinetic and mechanical aspects," J. Mater. Sci, vol. 29, no. 3, pp. 569-583, 1994.

[16] I. L. Hosier, A. S. Vaughan, and S. G. Swingler, "An investigation of the potential of ethylene vinyl acetate/polyethylene blends for use in recyclable high voltage cable insulation systems," J. Mater. Sci, vol. 45, pp. 2747-2759, 2010.

[17] J. B. Lambert, S. Gronert, H. F. Shurvell, D. Lightner, and R. G. Cooks, Organic Structural Spectroscopy, 2nd ed., Pearson Prentice Hall, 2011.

[18] F. H. Winslow, M. Y. Hellman, W. Matreyek, and S. M. Stills, "Autoxidation semicrystalline polyethylene," Polym. Eng. Sci., vol. 6, no. 3, pp. 273-278, 1966.
[19] S. F. Chabira, M. Sebaa, and C. G'sell, "Influence of climatic aging on the mechanical properties and the microstructure of low-density polyethylene films," J. Appl. Polym. Sci., vol. 110, pp. 2516-2524, 2008.

[20] B. Ranby and J. F. Rabek, Photodegradation, photo-oxidation and photo-stabilization of polymers, John Wiley \& Sons, 1975.

[21] A. S. Vaughan and G. C. Stevens, "On crystallization, morphology and radiation effects in poly (ether ether ketone)," Polym., vol. 36 no. 8, pp. 1531-1540, 1995

[22] F. Khabbaz, A. C. Albertsson, and S. Karlsson, "Chemical and morphological changes of environmentally degradable polyethylene films exposed to thermo-oxidation," Polym. Degrad. Stab., vol. 63, pp. 127-138, 1999.

[23] K. Anandakumaran and D. J. Stonkus, "Assessment of oxidative thermal degradation of crosslinked polyethylene and ethylene propylene rubber cable insulation," Polym. Eng. Sci., vol. 32, 1386-1393, 1992.

[24] W. Wang, T. Takada, Y. Tanaka, and S. Li, "Space charge mechanism of polyethylene and polytetrafluoroethylene by electrode/dielectrics interface study using quantum chemical method," IEEE Trans. Dielectr. Electr. Insul., vol. 24, no. 4, pp. 2599-2606, 2017.

[25] G. Chen, Y. Tanaka, T. Takada, and L. Zhong, "Effect of polyethylene interface on space charge formation," IEEE Trans. Dielectr. Electr. Insul., vol. 11, no. 1, pp. 113-121, 2004.

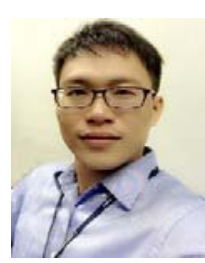

Somyot Tantipattarakul received a B.Eng. and M. Eng. degrees in Electrical Engineering from King Mongkut's University of Technology North Bangkok and Chulalongkorn University. He is now Engineer Lv.7 at Transmission system and Maintenance Department, Electricity Generating Authority of Thailand (EGAT) and $\mathrm{PhD}$ student at the University of Southampton.

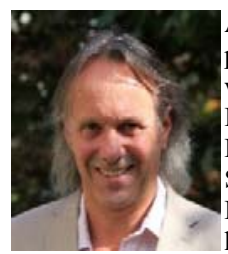

Alun Vaughan (SM'07) has a B.Sc. degree in chemical physics and a Ph.D. degree in polymer physics. After working at the UK's Central Electricity Research Laboratories and The University of Reading, he is now Professor of Dielectric Materials at the University of Southampton. He is a Fellow of the Institute of Physics, a Fellow of the IET and a Senior Member of IEEE. In 2016, he gave the IEEE DEIS Eric O. Forster Memorial Lecture.

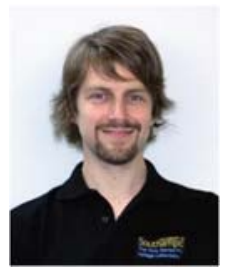

Thomas Andritsch (M'11) received the Dipl.-Ing. degree in electrical engineering from Graz University of Technology in 2006 and his $\mathrm{PhD}$ in the same field from Delft University of Technology in 2010. Since 2013 he has been a lecturer at the University of Southampton. He is currently a member of the IEEE DEIS AdCom, chair of the UK and Ireland Chapter of the IEEE DEIS and was member of IEEE and CIGRE working groups focusing on polymer-based nanodielectrics. 